\title{
ZEITSCHRIFT FÜR SPRACHWISSENSCHAFT
}

ORGAN DER DEUTSCHEN GESELLSCHAFT FÜR SPRACHWISSENSCHAFT

HERAUSGEBER

Deutsche Gesellschaft für

Sprachwissenschaft (DGfS)

\section{REDAKTION}

Federführung und Rezensionen

Sabine Arndt-Lappe

Universität Trier

Fachbereich II, Anglistik

54296 Trier

E-Mail: arndtlappe@uni-trier.de

\section{Stefanie Dipper}

Ruhr-Universität Bochum

Sprachwissenschaftliches Institut 44780 Bochum

E-Mail: dipper@linguistics.rub.de
Susann Fischer

Universität Hamburg

Institut für Romanistik

Von-Melle-Park 6

20146 Hamburg

E-Mail: susann.fischer@

uni-hamburg.de

Hans-Martin Gärtner

Hungarian Academy of Sciences

Research Institute for Linguistics

Benczúr u. 33

1068 Budapest

E-Mail: gaertner@nytud.hu

Gerhard Jäger

Universität Tübingen

Seminar für Sprachwissenschaft

Wilhelmstraße 19

72074 Tübingen

E-Mail: gerhard.jaeger@

uni-tuebingen.de 


\section{Gutachterrat}

Artemis Alexiadou (Berlin)

James Peter Blevins (Cambridge)

Daniel Büring (Wien)

Cornelia Ebert (Berlin)

Martina Faller (Manchester)

Paula Fikkert (Nijmegen)

Peter Gallmann (Jena)

Hubert Haider (Salzburg)

Katharina Hartmann (Frankfurt)

Nikolaus Himmelmann (Köln)

Roland Hinterhölzl (Venedig)

Anke Holler (Göttingen)

Baris Kabak (Würzburg)

Claudia Maienborn (Tübingen)
Theodoros Marinis (Konstanz)

Judith Meinschäfer (Berlin)

Christine Mooshammer (Berlin)

Natascha Müller (Wuppertal)

Petra Schulz (Frankfurt)

Elisabeth Stark (Zürich)

Christiane von Stutterheim (Heidelberg)

Benedikt Szmrecsanyi (Leuven)

Luka Szucsich (Berlin)

Judith Tonhauser (Ohio)

Ruben van de Vijver (Düsseldorf)

Ralf Vogel (Bielefeld)

Heike Wiese (Potsdam) 
ABSTRACTED/INDEXED IN Baidu Scholar · Cabell's Directory · Clarivate Analytics: Arts \& Humanities Citation Index; Journal Citation Reports/Social Sciences Edition; Social Sciences Citation Index; Web of Science · CNKI Scholar (China National Knowledge Infrastructure) · CNPIEC · De Gruyter: IBR (International Bibliography of Reviews of Scholarly Literature in the Humanities and Social Sciences); IBZ (International Bibliography of Periodical Literature in the Humanities and Social Sciences) $\cdot$ EBSCO (relevant databases) · EBSCO Discovery Service · Elsevier: SCOPUS · ERIH PLUS (European Reference Index for the Humanities and Social Sciences) $\cdot$ Gale/Cengage · Genamics JournalSeek · Google Scholar · J-Gate · JournalGuide · JournalTOCs · KESLI-NDSL (Korean National Discovery for Science Leaders) Linguistic Bibliography $\cdot$ Linguistics Abstracts Online $\cdot$ Microsoft Academic $\cdot$ MLA International Bibliography · Naviga (Softweco) · Primo Central (ExLibris) - ProQuest (relevant databases) · Publons · ReadCube $\cdot$ SCImago (SJR) $\cdot$ Sherpa/RoMEO $\cdot$ Summon (Serials Solutions/ProQuest) $\cdot$ TDNet $\cdot$ UB Frankfurt: BLL Bibliographie Linguistischer Literatur; OLC Germanistik; OLC Linguistik · Ulrich's Periodicals Directory/ulrichsweb · WanFang Data $\cdot$ WorldCat (OCLC)

ISSN 0721-9067 · e-ISSN 1613-3706

All information regarding notes for contributors, subscriptions, Open access, back volumes and orders is available online at www.degruyter.com/journals/zs

RESPONSIBLE EDITOR Stavros Skopeteas, Sprachwissenschaftliches Seminar, Georg-August-Universität, 37073 Göttingen, Email: stavros.skopeteas@uni-goettingen.de

ASSISTANT Susanne Trissler (Konstanz)

JOURNAL MANAGER Monika Wendland, De Gruyter, Genthiner Straße 13, 10785 Berlin, Germany, Tel.: +49 (0)30 260 05-190, Fax: +49 (0)30 260 05-250,

Email: monika.wendland@degruyter.com

RESPONSIBLE FOR ADVERTISEMENTS Claudia Neumann, De Gruyter, Genthiner Straße 13, 10785 Berlin, Germany, Tel.: +49 (0)30 260 05-226, Fax: +49 (0)30 260 05-264, Email: anzeigen@degruyter.com

(C) 2018 Walter de Gruyter GmbH, Berlin/Boston

TYPESETTING Meta Systems Publishing \& Printservices GmbH, Wustermark

PRINTING Franz X. Stückle Druck und Verlag e. K., Ettenheim

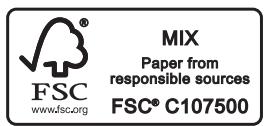





\section{Inhalt}

\section{Artikel}

Holden Härtl

Name-informing and distancing sogenannt 'so-called': Name mentioning and the lexicon-pragmatics interface - 139

Johannes Hein and Katja Barnickel

Replication of R-pronouns in German dialects - $\mathbf{1 7 1}$

\section{Das Linguistische Antiquariat}

Ralf Klabunde

Utz Maas: Grundkurs Sprachwissenschaft. Teil I: Die herrschende Lehre - 205 